\title{
IN-SITU SELF-ASSEMBLY OF SILICA NANOPARTICLES INTO MICROFIBERS WITH POTENTIAL TO REINFORCE POLYMERS
}

\author{
${ }^{1}$ Klára ZÁRYBNICKÁ, ${ }^{1}$ František ONDREÁŠ, ${ }^{1}$ Petr LEPCIO, ${ }^{2}$ Chao CHEN, ${ }^{1}$ Josef JANČÁŘ \\ ${ }^{1}$ Central European Institute of Technology, Brno University of Technology, Brno, Czech Republic, EU, \\ ${ }^{2}$ Polymer Science and Engineering, University of Massachusetts, United States of America, \\ klara.zarybnicka@ceitec.vutbr.cz
}

https://doi.org/10.37904/nanocon.2019.8489

\begin{abstract}
Silica nanosphere with a diameter of 10-15 nm were organized into fibers with a lenght of $15 \mathrm{~mm}$ and an aspect ratio of 100 by self-assembly in 1,4-dioxane. Dioxane causes a positive zeta potential on the silica surface thus silica in dioxane may behave as an acceptor (base catalyzer) causing decomposition of dioxane to acetaldehyde and its consequent polymerization into oligomer or polymer (polyoxyethylene) chains that bond the particles together. This process was proved using a thermogravimetric analysis which showed that the amount of polymerized dioxane is in the rang 2-3.5 wt. \%. Composition of the polymerized dioxane was elucidated employing FTIR. The formation of fibrillar structures was driven kinetically during solidification. The size of the fibers was controlled by the drying rate. Fast-drying results in longer and thinner fibers. Nanosilica fibers can also be formed in a polymer matrix (e.g. polycarbonate) via the solvent-casting method. Formation of fibers in-situ in a soft rubber polymer matrix in one-step processing can provide a polymer reinforcement at two hierarchical levels - at the nanoscale by immobilizing polymer chains due to the presence of nanoparticles and the microscale by strain transfer to the fibers. Elastic modulus of the fibers was determined by wrinkling technique by compression on the elastic surface and by thermal treatment in the polycarbonate matrix. Both techniques showed modulus 43-46 MPa.
\end{abstract}

Keywords: Self-assembly, fibers, nanosilica, dioxane

\section{INTRODUCTION}

Modern trends in the development of new materials seek inspiration in natural materials. Nature is able to create materials with outstanding properties from relatively simple components that are self-assembly arranged in a specific structure (e.g. a brick and mortar structure in a nacre that is made up of inorganic aragonite bound by a small amount of polymeric protein). Therefore, the bottom-up construction method (organization from the nanoscale, through micro and meso to macro dimensions) is increasingly being used in the preparation of synthetic materials. Nanoparticles and polymers thus become progressive building blocks nanoparticles cause mechanical reinforcement and can bring interesting functional properties (e.g. electrical conductivity, light emission) into the resulting nanocomposite. [1,2]

Many recent advances in the synthesis of self-assembly hybrid inorganic-organic materials have been reported. [3]. A common way of self-assembly nanocomposite preparation is mixing nanoparticles with a liquid monomer (followed by polymerization) or polymer solution (followed by evaporation of solvent). Nanoparticles can also be synthesized in-situ directly in a polymeric liquid. Solvent casting method is a powerful tool that can be used to easily control the arrangement of nanoparticles in a composite without the need for grafting or other surface treatments. The choice of solvent in the polymer-particle-solvent system changes the interactions of the individual components, thus arranging nanoparticles into defined structures - individual particles, chain bound clusters and contact aggregates. However, these self-assembly formations are randomly arranged (into statistically random or spherical formations), not showing anisotropy. [4,5] The arrangement of nanoparticles in the polymer has a crucial influence on the resulting properties of the composite. [6] 
The subject of our research is unique fibrous anisotropic structures that can be prepared from silica nanoparticles by a simple self-assembly solvent casting method in 1,4-dioxane. The result is a highly filled nanocomposite. These fibers can also be prepared in-situ in the polymer matrix where they can effectively reinforce the polymer matrix. The modulus of elasticity of silica fibers was determined to assess their ability to reinforce the composite.

One of the possible methods for determination of the elastic modulus $E$ of fine materials is wrinkling on the elastomeric substrate. $[7,8]$ This method is mainly used to determine $E$ of polymer films but has also been described for thin crystalline materials. The tested material is placed on an elastic substrate, most commonly polydimethylsiloxane (PDMS), with a known elastic modulus $E_{\mathrm{m}}$ and Poisson ratio $v$. A mechanical deformation is then applied to the substrate, which is transferred to the tested material caused its periodical wrinkling with a certain wavelength $\lambda$. With knowledge of the thickness of the tested material $h$, its elastic modulus $E_{\mathrm{f}}$ can then be determined according to equations (1). Good adhesion between the substrate and the test material is required.

$$
E_{\mathrm{f}}=3 \frac{E_{\mathrm{m}}}{1-v^{2}}\left(\frac{\lambda}{2 \pi h}\right)^{3}
$$

\section{MATERIALS AND METHODS}

Materials: Colloidal spherical silica pre-dispersed in $30.6 \mathrm{wt}$ \% isopropanol solution (IPA-ST, Nissan Chemicals). 1,4-dioxane in quality for analysis (Penta). Polycarbonate (PC) Macrolon $2407 \mathrm{C}$ with molecular weight $42 \mathrm{~kg} \cdot \mathrm{mol}^{-1}$ and polydispersity 2.2 (Bayer AG).

Sample preparation: Nanosilica was stirred in dioxane solution and then dried at an appropriate temperature on a tempered aluminum sheet (freestanding fibers preparation). PC was dissolved in dioxane, colloidal nanosilica was added, the solution was stirred and then dried on an aluminum sheet (nanocomposite preparation).

Characterization: Structural analysis were done employing scanning electron microscopy (SEM; samples covered with gold layer) and confocal laser microscopy (CLSM) for observation of freestanding fibers and transmission electron microscopy (TEM) for observation of ultramicrotome cuts of nanocomposites. Thermogravimetric analysis (TGA) were measured in a nitrogen atmosphere with temperature ramp $10^{\circ} \mathrm{C} \cdot \mathrm{min}^{-1}$ up to $650^{\circ} \mathrm{C}$. The silica sample was pressed into $\mathrm{KBr}$ tablets for infrared spectroscopy (FTIR). Determination of elastic modulus of the fibers was done by wrinkling technique on PDMS substrate (the surface and thickness of fibers were observed by optical profilometry, OP) and by thermal treatment compression in PC matrix.

\section{RESULTS AND DISSCUSION}

According to the trend of organization nano elements into larger structures using the bottom-up method, we have developed a method for organizing nanosilica particles of $10-15 \mathrm{~nm}$ up to $1.5 \mathrm{~cm}$ long fibers with aspect ratio 100 . These fibers are prepared by a self-assembly process in a 1,4-dioxane. Silica is capable to reach a positive partial charge on its surface in dioxane, which was verified by measuring $\zeta$-potential. Positively charged silica acts as alkali and catalyzed decomposition of dioxane to acetaldehyde and subsequent polymerization of acetaldehyde to oligomeric or polymeric (polyoxyethylene, POE) chains (Figure 1A). TGA showed that dioxane decomposes to a lower boiling substance such as acetaldehyde in the presence of silica (boiling point of dioxane is $101{ }^{\circ} \mathrm{C}$, of acetaldehyde $20^{\circ} \mathrm{C}$ ). TGA also showed a higher organic substance bound to the silica, which had a higher decomposition temperature (about $200^{\circ} \mathrm{C}$ ) than pure dioxane. The amount of the higher 
organic component in the fibers was 2-3.5 wt. \%. FTIR spectrum confirmed the presence of ether groups (e.g. typical strong peak at $1100 \mathrm{~cm}^{-1}$ ) present in the silica fibers that would correspond to POE polymerization.

It was suggested that dioxane polymerized and adsorbed on the silica surface acts as grafting short branching chains that control the arrangement of nanoparticles into anisotropic structures during solvent evaporation (dying of remaining original solvent, fiber solidification; Figure 1B). Rarely longer polymer chains can also serve as bridging molecules that are adsorbed on multiple particles simultaneously and interconnect them.

A

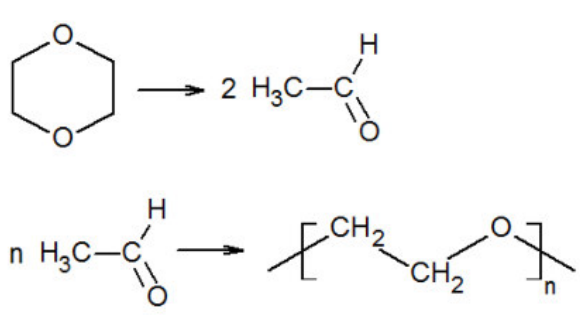

B

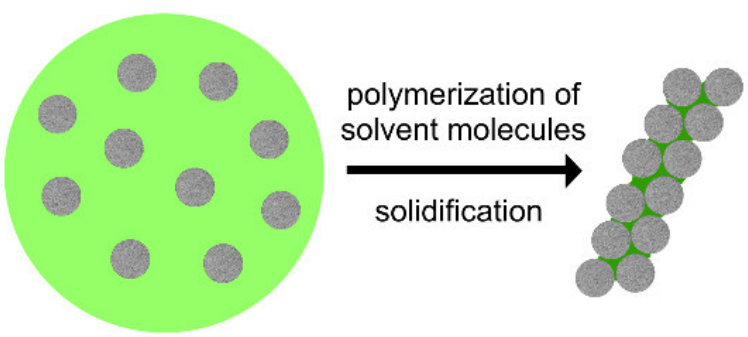

Figure $1 \mathrm{~A}$ - Scheme of 1,4-dioxane decomposition to acetaldehyde and its consecutive polymerization to POE. B - Illustration of the interconnection of nanoparticles into fibers (light green represents solvent dioxane, dark green represents polymerized dioxane)

Fibers were formed during solidification when solvent evaporated. No fiber formation was observed in the liquid solution after a long-time period ( 2 years). The kinetic of solidification also controls the morphology of the fibers - rapid drying at high temperatures led to long thin fibers with aspect ratio approximately 100 (width about $150 \mu \mathrm{m}$, length $1.5 \mathrm{~cm}$; Figure 2A, C), on the other hand, slow drying at low temperatures led to the formation of shorter and thicker fibers with an aspect ratio of about 20 (width $500 \mu \mathrm{m}$, length $1.0 \mathrm{~cm}$; Figure 2B). Longterm storage of silica in dioxane solution did not affect the content of polymerized dioxane bound to the silica surface after drying - the polymer content was the same whether the fibers were dries after 2 hours or after 6 months from dioxane solution. It can, therefore, be assumed that the silica surface was saturated with polymerized dioxane after stirring for 2 hours $\left(\right.$ at $\left.50^{\circ} \mathrm{C}\right)$. When the silica was solidified at a higher temperature after 6 months storage in solution, the same fibers were formed as when dried after 2 hours from mixing dioxane and nanosilica.
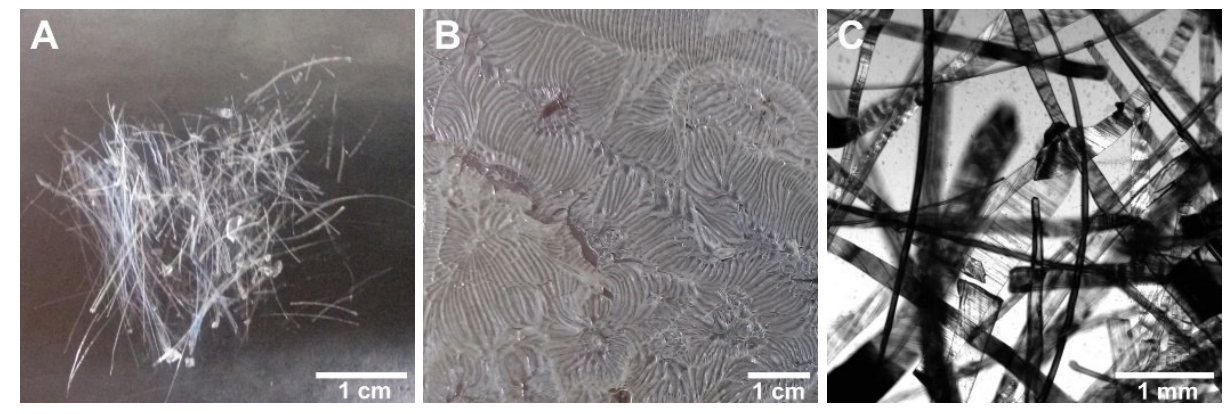

Figure 2 Free-standing silica fibers. A - Photo of rapidly dried fibers (drying temperature $150{ }^{\circ} \mathrm{C}$ ); B - photo of fibers dried at room temperature; $\mathrm{C}$ - detail of rapidly dried fibers (CLSM)

The fibers had a rectangular, almost square, cross-section, their surface was smooth without significant external structure (Figure 3A). Remarkable was the internal structure of the fibers, which was in the form of arcs. After drying of bulk material, the central backbone from which the individual arcs originated was observable (Figure 3B). When the solution was evaporated in a thin layer on a silicon wafer, very regular arcs were formed without a central backbone (Figure 3C). 

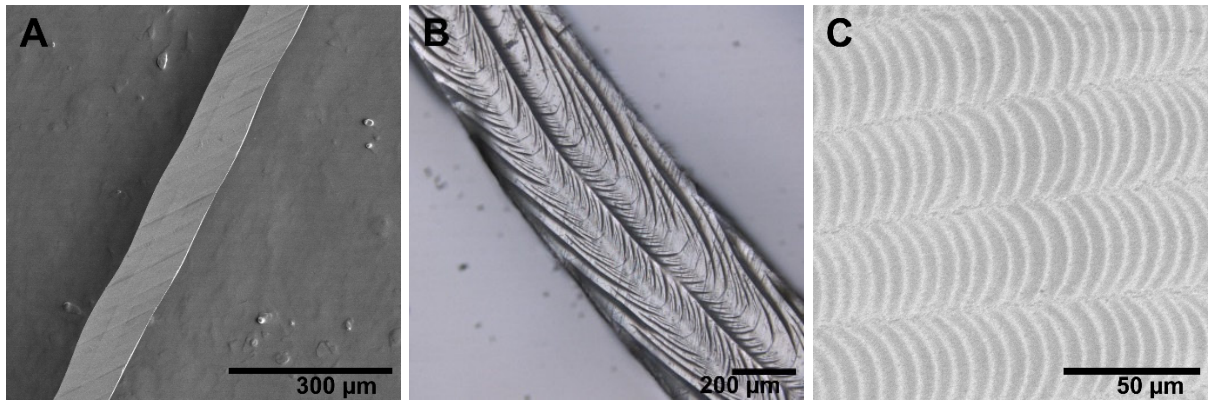

Figure 3 Structure of silica fibers. A - smooth surface (SEM); B - internal arc structure (CLSM);

$C$ - arc structure on fibers deposited in a thin layer on Si wafer (SEM)

The microfibers made of spherical nanosilica can also be prepared in-situ in the polymer matrix. The PC beads were dissolved in dioxane and nanosilica particles were added, the solution was again stirred at $50^{\circ} \mathrm{C}$ for 2 hours. Fibers shaped in the polymer matrix after solvent evaporation, but they were smaller than in freestanding version because the polymer chains slowed down the diffusion of nanoparticles and thus prevented the growth of fibers to macro dimensions during solidification. Best of our knowledge, no self-assembling organization of spherical nanosilica with the bare surface without grafting or other surface treatment into composites with anisotropic structures has been described in any other solvent.

Again, the size of the fibers and the structure of the nanocomposite can be controlled by the solidification kinetics - nanocomposites dried at 3 different temperatures are depictured in Figure 4 . When dried at $200{ }^{\circ} \mathrm{C}$, fibers with an average length of $2.5 \mu \mathrm{m}$ with an aspect ratio of $14 \pm 5$ were formed, at $150^{\circ} \mathrm{C}$ the fibers had a size of $5 \mu \mathrm{m}$ and an aspect ratio of $11 \pm 1$ and at $105^{\circ} \mathrm{C}$, slightly above the boiling point $\left(T_{\mathrm{B}}\right)$ of dioxane, fibers had an average size of $6 \mu \mathrm{m}$ and an aspect ratio of $13 \pm 4$. It should be noted that solidification close to the $T_{\mathrm{B}}$ of dioxane also produced large fibers of several tens of micrometers (Figure 4D), the fiber size distribution became wider with decreasing solidification temperature; the fibers showed the most uniform size when drying at high temperatures. In any case, it appeared that fibers of approximately the same aspect ratio are formed in the polymer matrix throughout the different solidification temperatures.
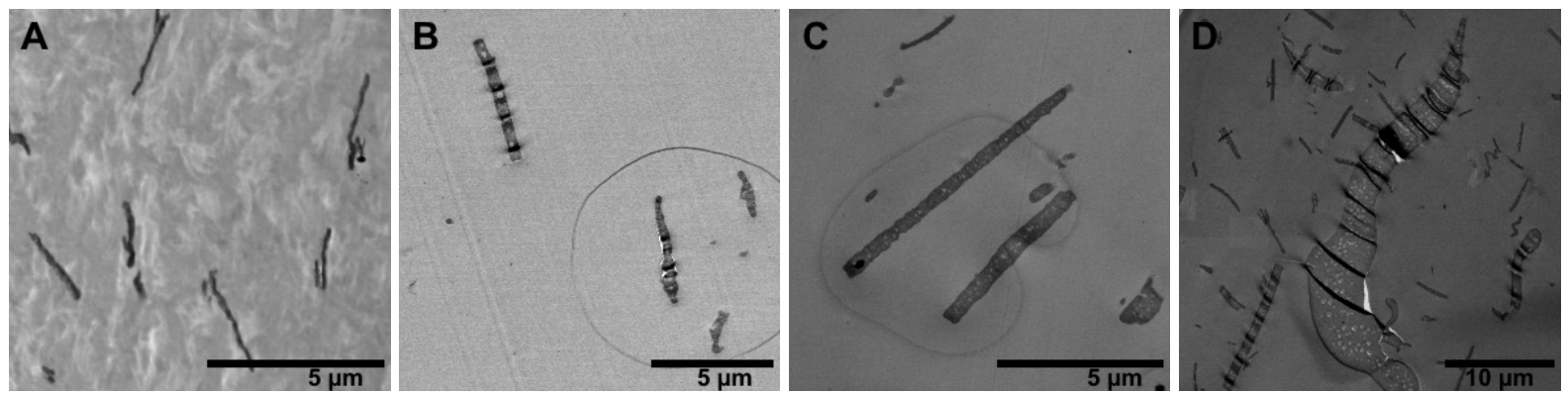

Figure 4 PC nanocomposites prepared in dioxane with 1 vol. \% of nanosilica dried at different temperature (TEM). A - high temperature $200{ }^{\circ} \mathrm{C} ; \mathrm{B}$ - medium temperature $150{ }^{\circ} \mathrm{C} ; \mathrm{C}$, D - low temperature $105^{\circ} \mathrm{C}$.

Anisotropic fibers in the polymer matrix have the potential to reinforce the matrix more than disordered aggregated nanoparticles. The reinforcing effect of nanoparticles has a contribution in substituting the volume of the soft matrix for hard particles and secondly the immobilization of polymer chains near the surface of the nanoparticles. Besides, the fibers can transfer stress and make the reinforcement even more efficient. Knowledge of fiber mechanical properties, in particular, the elastic modulus $E_{f}$, is essential to assess the possibility of fiber reinforcement of the polymer matrix. $E_{f}$ of free-standing fibers without polymer matrix was determined by wrinkling technique on a PDMS substrate (Figure 5A). The fibers from the silica were relatively brittle and did not allow large deformations, the fiber compression caused by the release of the prestressed 
substrate was $1-4 \%$. With the knowledge of the thickness of the fiber $h$, the elastic modulus of PDMS $E_{\mathrm{m}}$ (2 MPa), Poisson ratio of PDMS $v(0.46)$ and wavelength of wrinkles (Figure 5B) was determined the value of Ef to $43 \mathrm{MPa}$.
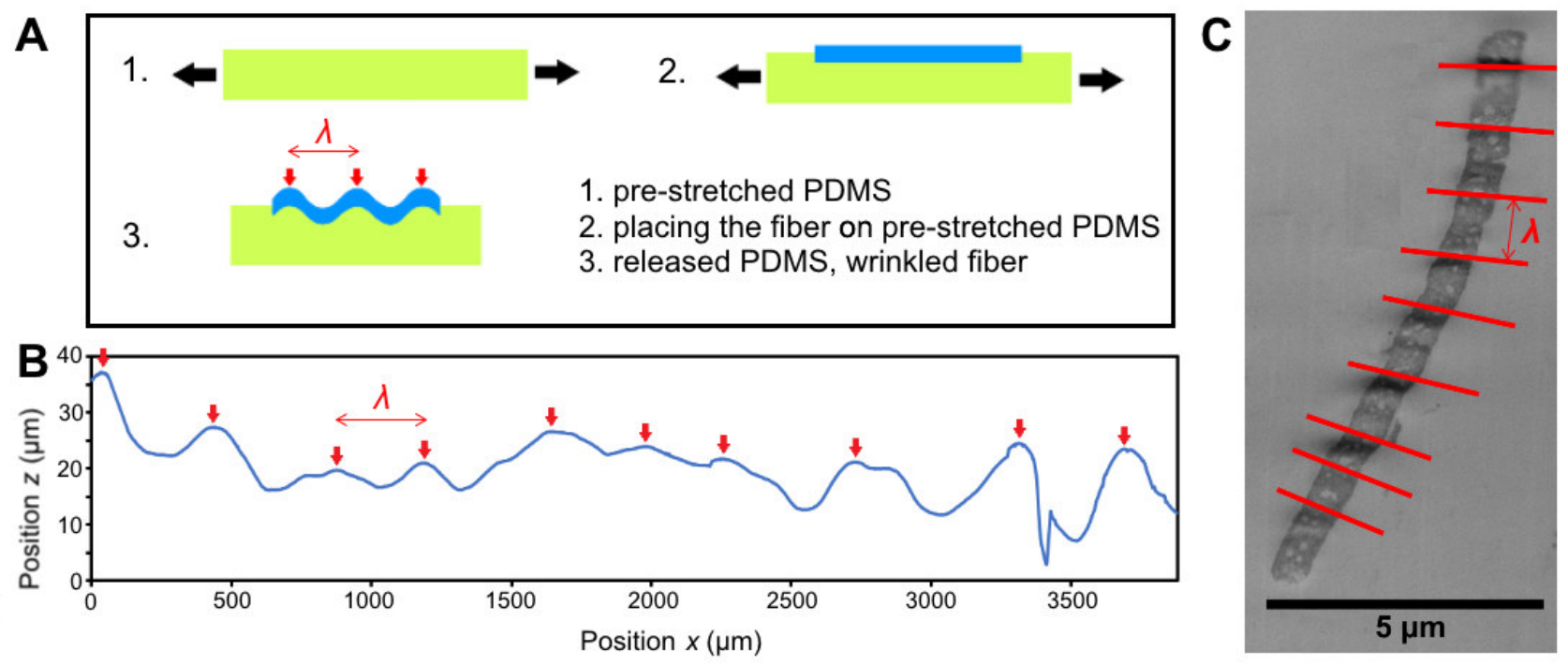

Figure 5 Determination of wrinkle wavelength $\lambda$. A - Scheme of wrinkling technique on the elastic substrate; $B$ - topology curve of silica fiber wrinkled on PDMS (OP); C - determination from TEM image after thermal treatment in $\mathrm{PC}$ matrix.

$E_{f}$ of fibers formed in-situ in the polymer matrix was determined by an alternative method, namely thermal treatment in the polymer matrix (Figure 5C). The fibers were formed during the solvent evaporation in a polymeric liquid (solution), which gradually passed to the polymer melt (initially softened by solvent molecules). When the nanocomposite was cooled rapidly to room temperature, the polymer solidified and shrinked, which again induced wrinkles on the fibers themselves. Similarly to the technique of wrinkling on the elastic substrate, the $E_{\mathrm{f}}=46 \mathrm{MPa}$ was calculated. Square cross-section of the fiber, the elastic modulus of PC $1.83 \mathrm{GPa}$ and Poisson ratio of PC 0.37 were taken into account.

Determination of $E_{f}$ by both techniques was in good correlation. From the $E_{f}$ value, it can be said that the mechanical properties of the fibers are determined by the elastic modulus of the organic component formed by the polymerization of dioxane. Bulk POE elastic modulus values are typically around 300 MPa, which would indicate that the POE adsorbed on the silica surface is not tightly bound between the particles or is composed of shorter oligomeric chains.

\section{CONCLUSION}

The tool for self-assembly organization of nanoparticles of $10-15 \mathrm{~nm}$ into microfibres with a length of $1.5 \mathrm{~cm}$ and an aspect ratio of 100 was shown. The key factor is the preparation of fibers in dioxane, which is capable to polymerize on nanosilica surface and control the arrangement of nanoparticles into anisotropic structures during solidification. The result is a highly filled composite, which mostly contains inorganic particles held together by 2-3.5 wt. \% of the organic component (oligomeric/polymeric POE). The preparation of the fibers is also possible in-situ in the polymer matrix, where the fibers could represent effective reinforcement. The elastic modulus of the fibers was determined by the wrinkling technique on an elastic substrate and confirmed by thermal induced wrinkling. The value of the elastic modulus of the fibers varied between 40-50 MPa giving them the ability to function as reinforcement in rubber polymers. 


\section{ACKNOWLEDGEMENTS}

Financial support under 18-17540S Grant from the Czech Grant Agency and CEITEC VUT-J-19-5962 Junior Grant from Central European Institute of Technology are appreciated. Part of this work was carried out with the support of CEITEC Nano Research Infrastructure (MEYS CR, 2016-2019).

\section{REFERENCES}

[1] WEGST, Ulrike G. K., BAI, Hao, SAIZ, Eduardo, TOMSIA, Antoni P. and RITCHIE, Robert O. Bioinspired structural materials. Natural Materials. 2015. vol. 14, no. 1, pp. 23-36.

[2] KAO, Joseph, THORKELSSON, Kari, BAI, Peter, RANCATORE, Benjamin J. and XU, Ting. Toward functional nanocomposites: taking the best of nanoparticles, polymers, and small molecules. Chem. Soc. Rev. 2013. vol. 42, no. 7 , pp. 2654-2678.

[3] CONG, Huai-Ping and YU, Shu-Hong. Self-assembly of functionalized inorganic-organic hybrids. Current Opinion in Colloid and Interface Science. 2009. vol. 14, no. 2, pp. 71-80.

[4] JOUAULT, Nicolas, ZHAO, Dan and KUMAR, Sanat K. Role of casting solvent on nanoparticle dispersion in polymer nanocomposites. Macromolecules. 2014. vol. 47, no. 15, pp. 5246-5255.

[5] LEPCIO, Petr, ONDREAS, Frantisek, ZARYBNICKA, Klara, ZBONCAK, Marek, CAHA, Ondrej and JANCAR, Josef. Bulk polymer nanocomposites with preparation protocol governed nanostructure: the origin and properties of aggregates and polymer bond clusters. Soft Matter. 2018, vol. 14, no. 11, pp. 2094-2103.

[6] ONDREAS, Frantisek, LEPCIO, Petr, ZBONCAK, Marek, ZARYBNICKA, Klara, GOVAERT, Leon. E. and JANCAR, Josef. Effect of nanoparticle organization on molecular mobility and mechanical properties of polymer nanocomposites. Macromolecules. 2019. vol. 52, no. 16, pp. 6250-6259.

[7] REYES-MARTINEZ, Marcos A., RAMASUBRAMANIAM, Ashwin, BRISENO, Alejandro L. and CROSBY, Alfred J. The instrinsic mechanical properties of rubrene single crystals. Advanced Materials. 2012. vol. 24, no. 41, pp. 5548-5552.

[8] INGUINIZ, Nestor, FRISENDA, Riccardo, BRATSCHITSCH, Rudolf and CASTELLANOS-GOMEZ, Andres. Revisitng the buckling metrology method to determine the Young's modulus of 2D materials. Advanced Materials. 2019. vol. 31, no. 10, pp. 1-6. 